\title{
POLYNOMIALS WITH NO SMALL PRIME VALUES
}

\author{
KEVIN S. MCCURLEY
}

\begin{abstract}
Let $f(x)$ be a polynomial with integer coefficients, and let

$$
D(f)=\text { g.c.d. }\{f(x): x \in \mathbf{Z}\} \text {. }
$$

It was conjectured by Bouniakowsky in 1857 that if $f(x)$ is nonconstant and irreducible over $\mathbf{Z}$, the $\mathbf{i}|f(x)| / D(f)$ is prime for infinitely many integers $x$. It is shown that there exist irreducible polynomials $f(x)$ with $D(f)=1$ such that the smallest integer $x$ for which $|f(x)|$ is prime is large as a function of the degree of $f$ and the size of the coefficients of $f$.
\end{abstract}

Let $f(x)$ be a polynomial with integer coefficients, and let $D(f)$ be the largest integer $D$ such that $D$ divides $|f(x)|$ for all integers $x$. It was conjectured by Bouniakowsky [B] in 1857 that if $f(x)$ is nonconstant and irreducible over the rationals, then $|f(x)| / D(f)$ is prime for infinitely many integers $x$. This conjecture is only known to be true in the case where $f(x)$ is of degree one, when Bouniakowsky's conjecture is equivalent to the well-known theorem of Dirichlet on primes in arithmetic progressions.

If Bouniakowsky's conjecture is true, then it seems natural to ask the question: How large is the smallest integer $x$ for which $|f(x)| / D(f)$ is prime? In the case where $f(x)$ is of degree one, an answer to this question is provided by a result of Linnik, that if $(a, q)=1$, then the least prime congruent to $a$ modulo $q$ does not exceed $q^{c_{1}}$. (In this note we use $c_{1}, c_{2}, \ldots$ to denote positive absolute constants.) On the other hand, it was proved by Prachar [P] that there exist positive integers $a$ and $q$ with $a<q$ and $(a, q)=1$ such that $a+q x$ is composite for all integers $x$ with

$$
0 \leqslant x \leqslant c_{2} \log q \log _{2} q \frac{\log _{4} q}{\left(\log _{3} q\right)^{2}},
$$

where $\log _{k} q$ is the $k$-fold iterated natural logarithm. In a previous paper by the author $[\mathbf{M}]$ a result was proved for polynomials of higher degree that is analogous to the result of Prachar. The purpose of the present note is to prove a stronger result of this type.

In order to provide a means to measure the size of the least $x$ for which $|f(x)| / D(f)$ is prime, we define the length $L(f)$ of a polynomial as follows.

DEFINITION. If $f(x)=\sum_{k=0}^{n} a_{k} x^{k}$ with $a_{k} \in \mathbf{Z}$, then $L(f)=\sum_{k=0}^{n}\left\|a_{k}\right\|$, where $\left\|a_{k}\right\|$ is the number of digits in the binary expansion of $a_{k}$, with $\|0\|=1$.

Received by the editors March 28, 1985.

1980 Mathematics Subject Classification (1985 Revision). Primary 11N32, 11R09. 
This definition is motivated by computer science concerns (see $[\mathbf{M}]$ ), but the result of this paper could be as easily formulated in terms of some other measure of the size of $f$, e.g. $L^{*}(f)=\log \left(\sum_{k=0}^{n} a_{k}^{2}\right)$.

In [M] it was proved that there exist irreducible polynomials $f(x)$ of arbitrarily large degree with $D(f)=1$ such that $|f(x)|$ is composite for all integers $x$ with

$$
|x|<\exp \left(\exp \left(c_{3} \frac{\log L(f)}{\log _{2} L(f)}\right)\right) \text {. }
$$

It was conjectured by Adleman and Odlyzko [AO] that if $f$ is irreducible, then the least $x$ for which $|f(x)| / D(f)$ is prime is $\ll \exp \left(L(f)^{c_{4}}\right)$. The following result shows that this conjecture is essentially best possible, since we must have $c_{4} \geqslant 1 / 2$.

THEOREM. There exist irreducible polynomials $f(x)$ of arbitrary degree with $D(f)=1$ such that $|f(x)|$ is composite for all integers $x$ with

$$
|x|<\exp \left(c_{5} \sqrt{L(f) / \log L(f)}\right) .
$$

The proof of this result is constructive and extremely simple, relying only on the Prime Number Theorem.

The principle behind the proof of this result is to choose $f(x)$ in such a way that the congruence $f(x) \equiv 0(\bmod p)$ has $p-1$ solutions modulo $p$ for all odd primes $p$ with $p-1 \leqslant n=$ degree of $f$. This forces the values of $x$ for which $|f(x)|$ is prime to belong to an arithmetic progression with modulus $\prod_{3 \leqslant p \leqslant n+1} p$.

Let $p_{1}<p_{2}<\cdots<p_{m}$ be the first $m$ odd primes, where $m$ is large. The following polynomials will be demonstrated to satisfy the claim made in the theorem of this note:

$$
f(x)=2 p_{1} \cdots p_{m}+\sum_{k=1}^{m-1} 2 p_{k+1} \cdots p_{m} \prod_{i=1}^{p_{k}-1}(x+2 i)+\prod_{i=1}^{p_{m}-1}(x+2 i) .
$$

Note that $f(x)=\sum_{k=0}^{n} a_{k} x^{k}$, where $a_{n}=1, n=p_{m}-1$, and $a_{k} \equiv 0(\bmod 2)$, $0 \leqslant k<n$. Furthermore we have $a_{0} \equiv 2(\bmod 4)$, so that $f(x)$ is irreducible by Eisenstein's Criterion.

Since $f(-2)=2 p_{1} \cdots p_{m}$ and $f(1)$ is odd, it follows that $D(f)$ divides $p_{1} \cdots p_{m}$. Note that if $1 \leqslant k \leqslant m$, then

$$
f(x) \equiv b_{k} \prod_{i=1}^{p_{k}-1}(x+2 i) \quad\left(\bmod p_{k}\right)
$$

for some integer $b_{k}$ with $b_{k} \not \equiv 0\left(\bmod p_{k}\right)$. Hence $f(x) \equiv 0\left(\bmod p_{k}\right)$ if and only if $x \not \equiv 0\left(\bmod p_{k}\right)$. From this it follows that $D(f)=1$. Furthermore, if $x$ is an integer with $0<|x|<p_{1} \cdots p_{m-1}$, then at least two of the primes $p_{1}, \ldots, p_{m}$ do not divide $x$, and it follows that $|f(x)|$ is composite. Since $f(0)$ is composite, we have established the fact that if $|f(x)|$ is prime, then $|x| \geqslant p_{1} \cdots p_{m-1}$.

We now estimate $L(f)$. Note that

$$
0 \leqslant a_{i} \leqslant f(1)<p_{m} !+m 2^{p_{m}} p_{m} !<p_{m} 2^{p_{m}} p_{m} !
$$


It follows from this and Stirling's formula that

$$
L(f) \leqslant p_{m}\|f(1)\|<c_{5} p_{m} \log p_{m} !<c_{6} p_{m}^{2} \log p_{m} .
$$

It now suffices to observe that from the Prime Number Theorem we obtain

$$
\log \left(p_{1} \cdots p_{m-1}\right)>c_{7} p_{m}>c_{8} \sqrt{L(f) / \log L(f)} .
$$

Observe that the polynomial $f(x)$ given here has degree $p_{m}-1$, but in fact there exist polynomials of every degree that satisfy Theorem 1. (If degree $n$ is desired and $p_{m} \leqslant n<p_{m+1}-1$, then it suffices to replace $(x+2)$ by $(x+2)^{n-p_{m}+2}$ in the definition of $f(x)$.) This improves the result of $[\mathbf{M}]$, where the degree $n$ had to be chosen from a very thin set.

\section{REFERENCES}

[AO] L. Adleman and A. Odlyzko, Irreducibility testing and factorization of polynomials, Math. Comp. 41 (1983), 699-709.

[B] V. Bouniakowsky, Sur les diviseurs numeriques invariables des fonctions rationelles entieres, Mem. Acad. Sci. St. Petersburg 6 (1857), 305-329.

[M] K. McCurley, Prime values of polynomials and irreducibility testing, Bull. Amer. Math. Soc. (N.S.) 11 (1984), 155-158.

[P] K. Prachar, Uher die Kleinste Primzahl einer arithmetischen Reihe, J. Reine Angew. Math. 206 (1961), 3-4.

Department of Mathematics, DRB 306, University of Southern California, Los Angeles, CALIFORNIA $90089-1113$ 